\title{
Ecofriendly dyeing of microwave treated cotton fabric using reactive violet $\mathrm{H} 3 \mathrm{R}$
}

\author{
Kiran S. ${ }^{1,}$, Adeel S. ${ }^{2}$, Rehman F.Ur. ${ }^{1}$, Gulzar T. ${ }^{1}$, Jannat M. ${ }^{1}$ and Zuber M. ${ }^{1}$ \\ 1Department of Applied Chemistry, Government College University Faisalabad 38000, Pakistan \\ 2Department of Chemistry, Government College University Faisalabad 38000, Pakistan \\ Received: 13/11/2017, Accepted: 13/11/2018, Available online: 21/11/2018 \\ *to whom all correspondence should be addressed: e-mail: shumaila.asimch@gmail.com \\ https://doi.org/10.30955/gnj.002523
}

\begin{abstract}
The urge of environmental friendly techniques in textile processing is gaining much attention due to high risks of effluent load causing eco-imbalance. For the present study, the cotton fabric and Reactive Violet $\mathrm{H} 3 \mathrm{R}$ dye solution were treated with microwave radiation for $2,4,6$, $8 \& 10 \mathrm{~min}$. at different powers (low, medium \& high). Dyeing was performed using un-irradiated (NRC) and irradiated cotton (RC) with un-irradiated (NRS) and irradiated dye solution (RS). Different dyeing parameters were optimized and ISO standard methods for color fastness were employed to rate the color change before and after irradiation. It is found that microwave irradiation for $8 \mathrm{~min}$. at high power has given good colour strength. When $60 \mathrm{~mL}$ irradiated dye solution of $7.5 \mathrm{pH}$ containing $8 \mathrm{~g} / \mathrm{L}$ of salt as exhausting agent is used to dye irradiated fabric at $60^{\circ} \mathrm{C}$ for $55 \mathrm{~min}$. ISO standards for colorfastness employed at various shades have shown that microwave irradiation has improved the rating of fastness properties. It is concluded that microwave irradiation has a promising potential to improve the color strength and dyeing properties under mild conditions.
\end{abstract}

Keywords: Cotton, reactive violet H3R dye, microwave radiation, sustainable tool, eco-friendly technique.

\section{Introduction}

The presence of toxic groups in dyes has raised environmental pollution and health problems (Bulut et al., 2012). Introduction of new eco-friendly techniques and bio-finishing of textile products have a promising effect on fabric processing to textile finishing (Tutak and Korkmaz, 2012; Saakshy et al., 2013). Researchers are trying to minimize the toxic effect of dyestuff and chemicals in the effluents by employing different techniques (Chatha et al., 2016; Iqbal et al., 2015a; lqbal et al., 2015b; Kiran et al., 2017) such as microwave (Al-Mousawi et al., 2013; Adeel et al., 2018), gamma (Zahid et al., 2017), ultrasonic (Tissera et al., 2016; Zeeshan et al., 2017) and UV treatment (Periolatto et al., 2014). These modern radiation techniques are being employed due to their cost, treatment, speed and energy effective nature (Ahmed and Shishtawy, 2014).

Of these techniques microwave irradiation is mostly used in textile industry due to its rapid mass transfer kinetics, which in turn takes less time, solvent \& energy consumption to give less time consumption leading to high treatment speed (Kale and Bhat, 2011). Being non-contact, uniform, and rapid heating source microwave irradiations are found as eco-friendly \& pollution free heating source for cotton dyeing (Hashem et al., 2014). It gives better color depth in short period as compared to conventional dyeing process. It also gives good dye exhaustion and better uptake ability through mass transfer effect (Haggag et al., 2014). Hence on account of the environmental friendly nature, the current study has been undertaken to make reactive dyeing of cotton using reactive violet $\mathrm{H} 3 \mathrm{R}$.

Reactive dyes produce brilliant and reproducible shades onto cotton fabrics (Nebojsa and Ivanka, 2012; Khatri et al., 2015). This is the only class of synthetic dyes that is capable of forming a covalent bond with the hydroxyl group of cellulosic fabric at given conditions (Patel et al., 2014; Abdel-Thalouth et al., 2014). Cotton (cellulosic fiber) the main substrate in textile world, is an ester linked chains made up of micro fibrils contain $94 \%$ of cellulose. Hydroxyl group present in its cellulosic structure is responsible for covalent linkage with the reactive group of reactive dyes (Figure 1). This exclusive property of bonding with dye molecule represents its dye uptake ability (Adeel et al., 2017a, 2017b). Researchers are modifying the surface of cotton fabric (Fang et al., 2013) to enhance its uptake ability through various tools including microwave treatment (Wojnarovist et al., 2010; Mohsin et al., 2013).

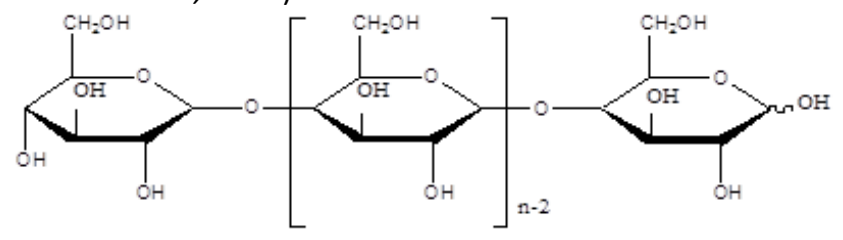

Figure 1. Structure of cellulose (cotton) 
Keeping in view of the advantages of microwave in textile processing, the current study was carried out to employ the microwave radiation onto cotton fabric using Reactive Violet H3R dye. The objective of this study is to improve color strength and color fastness properties of fabrics dyed with Reactive Violet H3R dye.

\section{Materials and methods}

\subsection{Sample preparation and irradiation}

Reactive Violet H3R ( $\mathrm{Cl} 182130)$ commonly known as Reactive Violet I (Figure 2) was obtained from Sohaib Dyes and Chemicals (Pvt.), Faisalabad, Pakistan. Plain weaved, mercerized and bleached cotton was provided by Noor Fatima Fabrics (Pvt.) Faisalabad, Pakistan. Both cotton fabric and dye solutions were irradiated for 2, 4, 6, 8 and $10 \mathrm{~min}$. at high, medium and low powers using commercial microwave oven Orient (Model: OM720CXNB; Rated Voltage: 230V; Rated Frequency: $50 \mathrm{~Hz}$ ). The dyeing of un-irradiated (NRC) \& irradiated cotton (RC) using irradiated \& unirradiated by keeping volume material to liquor ratio of dye bath [1:30].<smiles>COc1cc(Cl)c(O)c(/N=N/c2c([N+](=O)[O-])cc3cc(S(C)(=O)=O)cc(Nc4nc(Cl)nc(Nc5cccc(S(C)(=O)=O)c5)n4)c3c2O)c1</smiles>

Figure 2. Reactive violet $\mathrm{H} 3 \mathrm{R}(\mathrm{Cl} 182130)$

\subsection{Optimization of coloring conditions}

The effect of microwave irradiation was investigated by optimizing different dyeing variables keeping other parameters constant. Dyeing of optimal fabric was carried out using cotton fabric for 15, 25, 35, 45, 55 and 65 minutes, at 7, 7.5, 8, 8.5, 9, 9.5, 10, 10.5, 11 and 11.5 $\mathrm{pH}$ to acquire maximum exhaustion, 2, 4, 6, 8 and $10 \mathrm{~g} / \mathrm{L}$ of $\mathrm{NaCl}$ and $\mathrm{Na}_{2} \mathrm{SO}_{4}$ separately were employed. Finally, material to liquor ratio (M:L) 20, 30, 40, 50, 60, $70 \& 80$ $\mathrm{mL}$ of dye solutions were used to dye $1 \mathrm{~g}$ of fabrics (Yang et al., 2017).

\subsection{Evaluation of quality characteristics of dyed fabric}

All un-irradiated and irradiated dyed fabrics were investigated to find out $(\mathrm{K} / \mathrm{S})$ color strength using spectraflash SF 600 equipped with an illuminant D6510 observer at Department of Applied Chemistry, Govt. College University Faisalabad, Pakistan. Standard methods for color fastness to dry cleaning ISO 105 DO1, for color fastness to perspiration ISO 105 EO4. Wash fastness to ISO 105 CO3 for crocking (rubbing) ISO 105 X12 protocol and for Light fastness ISO 105 BO2 in fadometer has been employed (Adeel et al., 2015).

\section{Results and discussion}

The data given in Figure $3(a, b, c)$ reveal that microwave treatment at high power to both dye solution (RS) and cotton fabric (RC) for $8 \mathrm{~min}$. has given good colour depth $(\mathrm{K} / \mathrm{S})$ and darker shades as compared to fabric dyed with reactive dye solution under medium and low power microwave irradiation. Microwave irradiation create polarity \& volumetric heating inside the solution bonding with of cotton fabric (Adeel et al., 2016). Irradiation for low time do not stimulate the colorant to such towards the fabric surface, where for high time heating may degrade the colorant \& by-product formed after degradation get significant chance to sorb onto fabric, which in turn gives low $\mathrm{K} / \mathrm{S}$ value. Hence microwave irradiation for $8 \mathrm{~min}$. at high power to both dye solution (RS) and cotton fabric (RC) is recommended to get darker shades and good fastness properties.

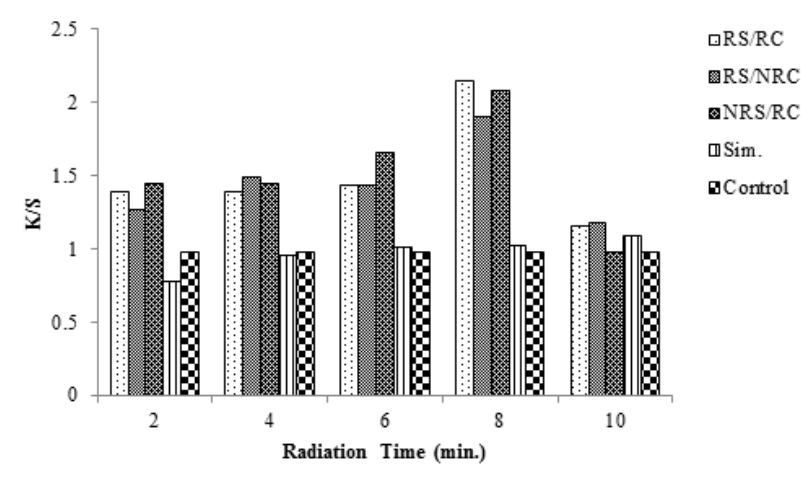

Figure 3(a). Effects of microwave treatment time at high power on dyeing of the cotton fabric using Reactive Violet H3R dye

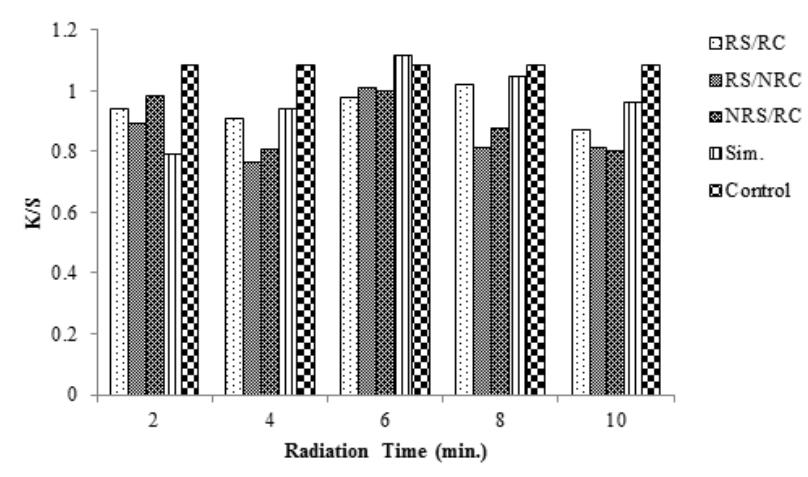

Figure 3(b). Effects of medium power microwave treatment time at medium power on dyeing of the cotton fabric using Reactive Violet H3R dye

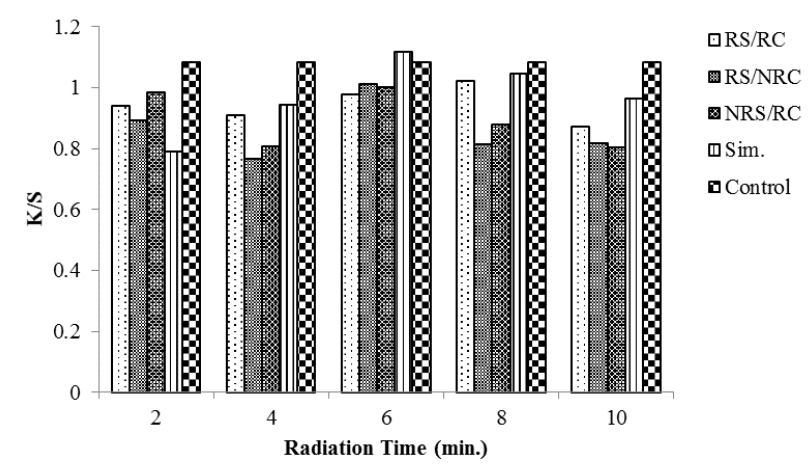

Figure 3(c). Effects of microwave treatment time at low power on dyeing of the cotton fabric using Reactive Violet H3R dye 
Salt being an exhausting agent always plays an important role in exhaustion of dye bath. It is found that addition of optimum amount of salt/electrolyte stimulates dye uptake that enhances the substantively but the exceed concentration of salt contributes to over exhaustion which lead towards unevenness (Teng et al., 2010). On the other hand, lower amount of electrolyte does not cause significant exhaustion during dyeing which gives less color depth (K/S) (Singha et al., 2013). Only the amount of salt added creates the dye bath environment to make significant attraction of dye with fabric within short range of forces (Tania and Millat, 2017). Thus $8 \mathrm{~g} / \mathrm{L}$ is the optimum amount of salt to achieve maximum exhaustion (Figure 4) for getting good color strength.

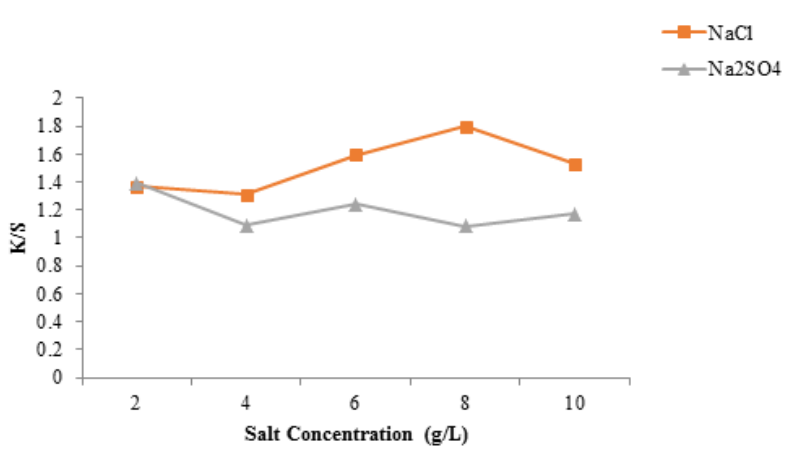

Figure 4. Effect of salt concentration on dyeing of irradiated cotton using irradiated dye solution

The role of dye bath volume is very important in reactive dyeing. It is found Figure 5 that $\mathrm{MW}$ treatment (RS/RC $8 \mathrm{~min}$.) to both dye solution and cotton fabric has reduced the amount of dye bath. Low dye bath volume gives less color strength, while high dye bath volume causes over sorption of dye molecules onto irradiated cotton fabric (Miljkovic et al., 2007). Using $60 \mathrm{~mL}$ of irradiated dye solution (RS) cause even sorption onto irradiated fabric $(\mathrm{RC})$ results into maximum color strength $(\mathrm{K} / \mathrm{S})$ \& darker shades.

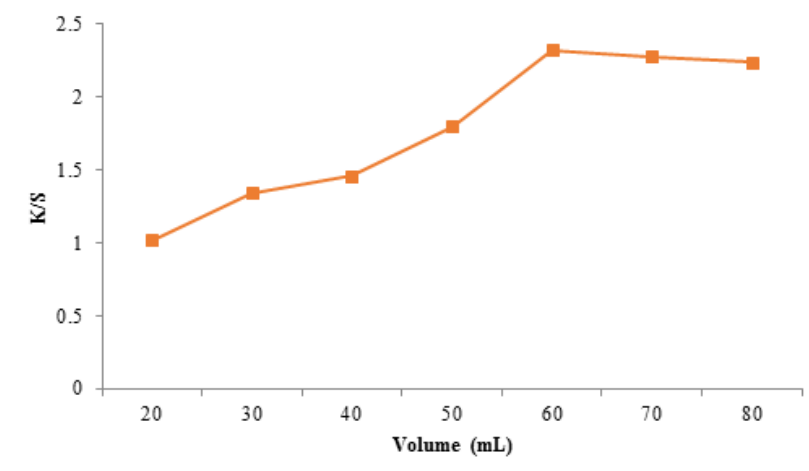

Figure 5. Effect of volume of dye bath on dyeing of irradiated cotton using irradiated dye solution

Dyeing time is an important factor in reactive dyeing using irradiated solution (RS) on irradiated cotton fabric (RC). Optimum dyeing time is considered to achieve maximum sorption and high color depth (Adeel et al., 2016).
However, lower dyeing time does not allow enough sorption of dye onto cotton fabric. Leveled dyeing and maximum strength is achieved by dyeing irradiated cotton fabric for $55 \mathrm{~min}$. that leads to high substantivity (Figure 6). Hence $55 \mathrm{~min}$. is recommended time for getting darker shades \& excellent color characteristics.

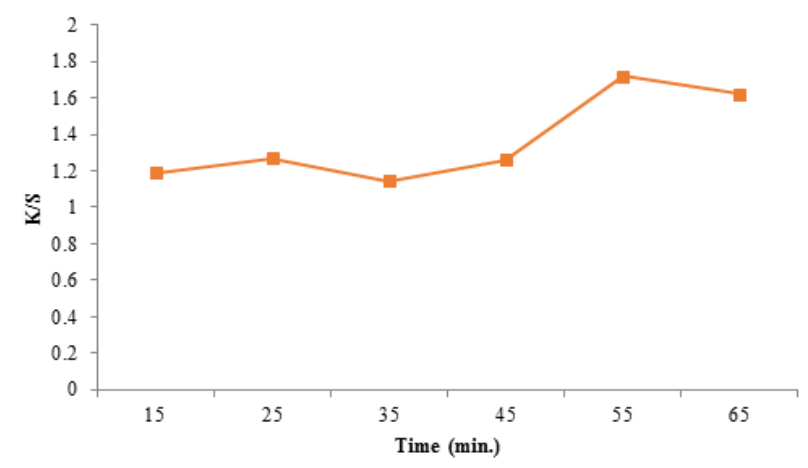

Figure 6. Effect of time on dyeing of irradiated cotton using irradiated dye solution

Temperature is one of the important parameters in reactive dyeing. This is because upon gradual rise of temperature, the kinetic energy of colorant is raised and its sorption ability is enhanced, thereby giving excellent color strength on to fabrics. Low heating level does not rise kinetic energy, whereas, high heating level may shift the dye bath equilibrium from fabric to solution which in turns gives low color strength (Clark, 2011). Hence after microwave treatment, $60{ }^{\circ} \mathrm{C}$ is the optimum temperature for coloration of irradiated fabric to achieve good color characteristics (Figure 7).

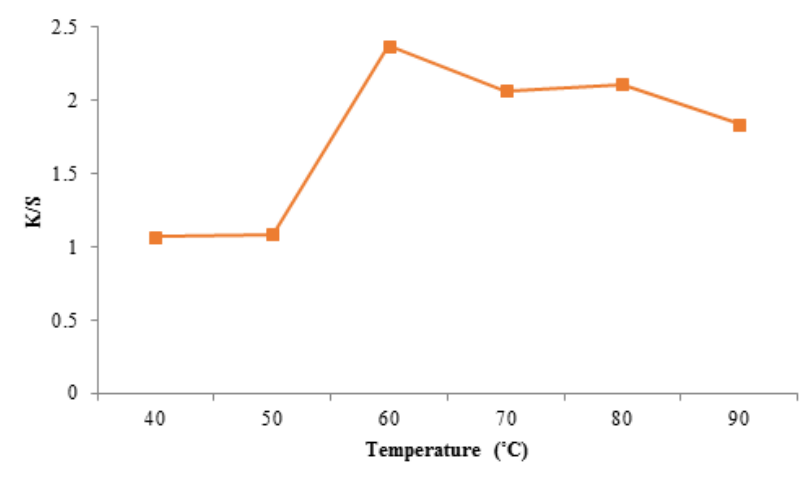

Figure 7. Effect of temperature on dyeing of irradiated cotton using irradiated dye solution

Reactive dyeing is $\mathrm{pH}$ dependent because mostly reactive dyeing occurs in alkaline media. At optimum $\mathrm{pH}$ of (7.5), leveled dyeing is achieved, which in turn gives high $\mathrm{K} / \mathrm{S}$ value (Lewis and Vo, 2007; Fu et al., 2013). Hence $\mathrm{pH} 7.5$ (Figure 8) almost neutral condition is favored dyeing of cotton under the influence of microwave treatment.

The rating results given in Table 1 show MW treatment has improved the colorfastness properties of dyed cotton fabrics. Irradiated fabrics were dyed using irradiated solution of $1-2.5 \%$ at optimal conditions. Enhanced/improved color fastness properties are due to 
the microwave irradiation as these irradiations have modified the surface of cotton. Wash fastness showed good to better results because of the strong linkage between primary hydroxyl group of cellulosic cotton fabric and reactive group of reactive dye that don't allow the dye molecule to detach from the fabric surface (Kim et al., 2014; Bhatti et al., 2016). This strong covalent bonding also resists when the fabrics were exposed to light, rubbing and dry cleaning. Modification in cotton surface has improved the dyeing behavior of fabric. It was found to be significant because upon exposure to light less fading of shades was observed. The dye structure has benzene ring due to which conjugation has improved the perspiration fastness. Hence, microwave irradiation has improved the fastness properties of cotton fabrics dyed with Reactive Violet H3R dye.

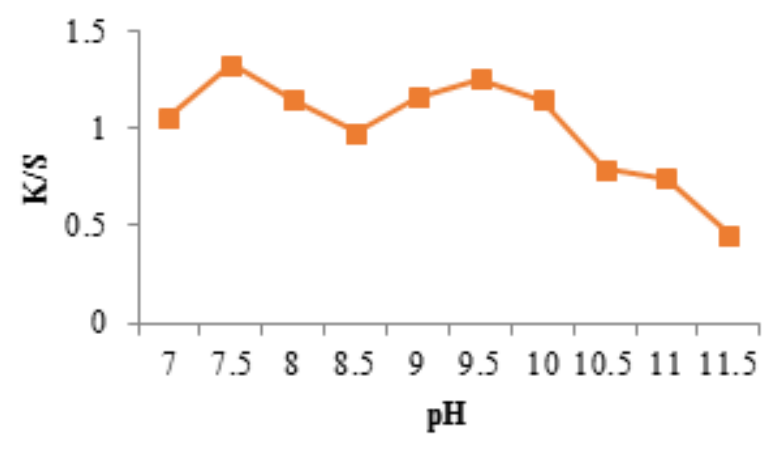

Figure 8. Effect of $\mathrm{pH}$ on dyeing of irradiated cotton using irradiated dye solution

Table 1. Effect of microwave treatment on color fastness properties of various shades of dyed fabric

\begin{tabular}{ccccccccc}
\hline \multirow{2}{*}{$\begin{array}{c}\text { Dye } \\
\begin{array}{c}\text { Conc. } \\
(\%)\end{array}\end{array}$} & Dry cleaning & \multicolumn{2}{c}{ Perspiration } & \multicolumn{2}{c}{ Rub fastness } & \multicolumn{2}{c}{ Wash fastness } & Light \\
\cline { 2 - 9 } & Acid & Alkaline & Dry & Wet & $\begin{array}{c}\text { Colour } \\
\text { change }\end{array}$ & $\begin{array}{c}\text { Colour } \\
\text { Stain }\end{array}$ & Fastness \\
\hline 0.1 & $3-4$ & $3-4$ & $3-4$ & 3 & $3-4$ & 3 & 3 & $3-4$ \\
\hline 0.5 & 4 & $4-5$ & $4-5$ & $3-4$ & 3 & $4-5$ & $4-5$ & $4-5$ \\
\hline 1 & $4-5$ & $4-5$ & $4-5$ & 4 & 4 & $4-5$ & $4-5$ & 4 \\
\hline 1.5 & 4 & 4 & $4-5$ & 5 & 5 & $3-4$ & $4-5$ & $3-4$ \\
\hline 2 & 4 & 4 & $4-5$ & $4-5$ & 5 & 3 & 4 & $3-4$ \\
\hline 2.5 & $4-5$ & $4-5$ & $4-5$ & 4 & $3-4$ & $3-4$ & $4-5$ & 4 \\
\hline
\end{tabular}

\section{Conclusions}

The current study has been conducted to investigate the effect of microwave treatment on reactive dyeing of cotton fabric using Reactive Violet H3R dye. Being uniform and rapid heating source the irradiated solution for $8 \mathrm{~min}$. at high power has enhanced the colour depth by dyeing un-irradiated cotton fabric at $60{ }^{\circ} \mathrm{C}$ for $55 \mathrm{~min}$. using $60 \mathrm{ml}$ of $7.5 \mathrm{pH}$ dye bath using $60 \mathrm{ml}$ in presence of $8 \mathrm{~g} / \mathrm{L}$ salt as exhausting agent. ISO standards applied for colour fastness upon rating show that microwave treatment has did not lower the results. The reduction in time of dyeing and salt amount proves that it is cost and time effective tool and makes the process eco-friendly. Hence this radiation tool can successfully be applied onto modification of different application methods of dyeing using natural and synthetic fabrics.

\section{Acknowledgements}

We are grateful to Higher Education Commission of Pakistan (HEC) for funding the project (No. 20-2724/NRPU/R\&D/ $\mathrm{HEC} / 12 / 6828$ ). We are also thankful to Mr. Zafar Iqbal Manger QA \& QC of Noor Fatima Textile (Pvt.) Limited, Faisalabad Pakistan and Mr. Muhammad Abbas Director Harris Dyes and Chemical (Pvt.) Limited, Faisalabad for providing the technical assistance and implementation of the work at industrial scale.

\section{References}

Abdel-Thalouth I., Ragheb A.A., Rekaby M., El-Hennawi H.M., Shahin A.A. and Haggag K. (2014), Application of microwave in textile printing of cellulosic fabrics, Research Journal of Chemical Sciences, 4, 41-46.
Adeel S., Usman M., Haider W., Saeed M., Muneer M. and Ali M. (2015), Dyeing of gamma irradiated cotton using Direct Yellow 12 and Direct Yellow 27: improvement in colour strength and fastness properties, Cellulose, 22, 2095-2105.

Adeel S., Ghaffar A., Mushtaq M., Yameen M., Rehman F., Zuber M., Kamran M. and lqbal M. (2016), Bio-processing of Surface oxidized cellulosic fibre by microwave treatment for eco-friendly textile dyeing, Oxidation Communication, 39, 2222-2396.

Adeel S., Kiran S., Ayesha T., Rehman F., Gulzar T., Rafi S., Zuber M. (2017a), Radiation pre-treatment: A potential novel technology to improve fastness properties of plant derived natural dyes, Wiley Scrivener Publishing LLC USA, 1, 65-87.

Adeel S., S Kiran., T Gulzar., Rehman F., Azeem M., Ahmad Z., Zuber M., Kamran M., Iqbal Z. (2017b), Influence of ultrasonic radiation on the dyeing of cotton fabric using Reactive Yellow 145 Dye, J. Natural Fibers, 14,658-665.

Adeel S., Zuber M., Rehman F., Zia K.M. (2018), Microwaveassisted extraction and dyeing of chemical and bio-mordanted cotton fabric using harmal seeds as a source of natural dye, Environmental Science and Pollution Research, 25, 11100-11110.

Ahmed N.E. and Shishtawy E.R. (2014), The use of new technologies in coloration of textile fibers, Journal of Material Sciences, 45, 1143-1153.

Al-Mousawi M.S., El-Apasery A.M. and Elnagdi H.M. (2013), Microwave assisted dyeing of polyester fabrics with disperse dyes, Molecules, 18, 11033-11043.

Bhatti I.A., Adeel S., Perveen S., Zuber M. (2016), Dyeing of UV irradiated cotton and polyester fabrics with multifunctional reactive and disperse dyes, Journal of Saudi Chemical Society, 20(2), 178-184. 
Bulut M.O. and Akar E. (2012), Ecological dyeing with some plant pulps on woollen yarn and cationized cotton fabric, Journal of Cleaner Production, 32, 1-9.

Chatha S.A.S., Kiran S., Gulzar T., Kamal S., Ghaffar A. and Chatha M.N. (2016). Comparative study on decolorization and mineralization of synthetic and real textile effluents using advanced oxidation processes, Oxidation Communications, 39(2), 1604-1614.

Clark M. (2011), Handbook of textile and industrial dyeing: principles, processes and types of dyes, vol. 1. Cornwall, UK, Woodhead Publishing, 1, 156-182.

Fang L., Zhang X. and Sun D. (2013), Chemical modification of cotton fabrics for improving utilization of reactive dyes, Carbohydrate Polymers, 91, 363-369.

Fu S., Hinks D., Hauser P. and Ankeny M. (2013), High efficiency ultra-deep dyeing of cotton via mercerization and cationization, Cellulose, 20, 3101-3110.

Haggag K., El-Molla M.M. and Mahmoued Z.M. (2014), Dyeing of cotton fabrics using reactive dyes by microwave irradiation technique, Indian Journal of Fibre and Textile Research, 39, 406-410.

Hashem M., Taleb A.M., El-Shall FN. and Haggag K. (2014), New prospects in pretreatment of cotton fabrics using microwave heating, Carbohydrate Polymers, 103, 385-391.

Iqbal M. and Nisar J. (2015), Cytotoxicity and mutagenicity evaluation of gamma radiation and hydrogen peroxide treated textile effluents using bioassays, Journal of Environmental Chemical Engineering, 3, 1912-1917.

Iqbal M., Abbas M., Arshad M., Hussain T., Khan A.U., Masood N., Tahir M.A., Hussain S.M., Bokhari T.H. and Khera R.A. (2015), Gamma radiation treatment for reducing cytotoxicity and mutagenicity in industrial wastewater, Polish Journal of Environmental Studies, 24, 2745-2750.

Kale M.J. and Bhat N.V. (2011), Effect of microwave pretreatment on the dyeing behaviour of polyester fabric, Coloration Technology, 127, 365-371.

Khatri A., Peerzada M.H., Mohsin M. and White M. (2015), A review on developments in dyeing cotton fabrics with reactive dyes for reducing effluent pollution, Journal of Cleaner Production, 87, 50-57.

Kim M.R., Kim H. and Lee J.J. (2014), Dyeing and fastness properties of vat dyes on meta-aramid woven fabric, Fibers and Polymers, 14, 2038-2044.

Kiran S., Adeel S., Nosheen S., Hassan A., Usman M., Rafiqu M.A. (2017), Recent trends in textile effluent treatments: A review, Advanced Materials for Wastewater Treatment, 1, 29-49.

Lewis D.M. and Vo L.T.T. (2007), Dyeing cotton with reactive dyes under neutral conditions, Coloration Technology, 56, 1478-4408.

Miljkovic M.N., Ignjatovic V.B. and Zarubica A.R. (2007), Influence of Different Parameters on Dyeing of Knitting Material with Reactive Dye, Physics, Chemistry and Technology, 5, 69-84.

Mohsin M., Rasheed A., Farooq A., Ashraf M. and Shah A. (2013), Environment friendly finishing of sulphur, vat, direct and reactive dyed cotton fabric, Journal of Cleaner Production, 53, 341-347.

Nebojsa R. and Ivanka R. (2012), Cationic Modification of Cotton Fabrics and Reactive Dyeing Characteristics, Journal of Engineered Fibers and Fabrics, 7, 113-121.
Patel D.R., Patel B.M., Patel N.B. and Patel K.C. (2014), Application of newly synthesized bisazodichloro-s-triazinyl reactive dyes bearing 1, 3, 4-oxadiazole molecule, Journal of Saudi Chemical Society, 18, 245-254.

Periolatto M., Ferrero F. and Migliavacca G. (2014), Low temperature dyeing of wool fabric by acid dye after UV irradiation, Journal of Textile Institute, 105, 1058-1064.

Saakshy A.K., Sharma K. and Jain R.K. (2013), Application of natural dyes: an emerging environment-friendly solution to handmade paper industry, Biotechnology for Environmental management and Resource Recovery, 15, 279-288.

Singha K., Maity S. and Singha M. (2013), The salt-free dyeing on cotton: an approach to effluent free mechanism; can chitosan be a potential option, International Journal of Science Education, 1, 69-77.

Tania A. and Millat M.A.K.M. (2017), Salt Free Dyeing of Cotton Fiber-A Critical Review, International Journal of Textile Sciences, 6, 21-33.

Teng X., Ma W. and Zhang S. (2010), Application of tertiary amine cationic poly acryl amide with high cationic degree in salt-free dyeing of reactive dyes, Chinese Journal of Chemical Engineering, 18, 1023-1028.

Tissera N.D., Ruchira N., Wijesena K.M. and Nalin D.S. (2016), Ultrasound energy to accelerate dye uptake and dye-fiber interaction of reactive dye on knitted cotton fabric at low temperatures, Ultrasonics Sonochemistry, 29, 270-278.

Tutak M. and Korkmaz N.E. (2012), Environmentally friendly natural dyeing of organic cotton, Journal of Natural Fibers, $\mathbf{9}$, 51-59.

Wojnarovits L., Foldvary C. and Takacs M.E. (2010), Radiation induced grafting of cellulose for adsorption of hazardous water pollutants: a review, Radiation Physics and Chemistry, 79, 848-857.

Yang D., Kong X., Gao D., Cui H., Huang T. and Lin J. (2017), Dyeing of cotton fabric with reactive disperse dye contain acyl fluoride group in supercritical carbon dioxide, Dyes and Pigments, 139, 566-574.

Zahid M., Bhatti I.A., Adeel S. and Saba S. (2017), Modification of cotton fabric for textile dyeing: industrial mercerization versus gamma irradiation, Journal of Textile Institute, 108, 287-292. 